\title{
Maxima Quaestio. El proyecto de un sistema definitivo según G.W. Leibniz
}

\author{
Maxima Quaestio. \\ The proyect of a definitive system \\ according to G.W. Leibniz
}

\author{
MIGUEL PALOMO \\ Universidad de Sevilla
}

Recibido: 15/12/2017 Aceptado: 19/12/2017

\section{RESUMEN}

En la modernidad, filósofos y científicos comenzaron a crear nuevas formas de explicar la naturaleza y mediante ello rompieron lazos con la filosofía griega. Leibniz, sin embargo, no solamente reconocía su influencia griega, sino que además ésta fue esencial en el desarrollo de un sistema definitivo de verdades, el cual hemos denominado como maxima quaestio.

PALABRAS CLAVE

LEIBNIZ, METAFÍSICA, MÓNADAS, MATEMATIZACIÓN DE LA NATURALEZA.

\section{ABSTRACT}

In the modern era, philosophers and scientists started to create new explanatory systems. By doing so, they were breaking ties with the past, especially with the Greek tradition. However, the case of Leibniz is different. First, he recognized the influence of the Greeks in his philosophy; and second, this influence was essential for the development of a definitive system of truths, which we have called the maxima quaestio.

KEYWORDS

LEIBNIZ, METAPHYSICS, MONADS, MATHEMATIZATION OF NATURE.

(C) Contrastes. Revista Internacional de Filosofia, vol. XXII-N² (2017), pp. 93-105. ISSN: 1136-4076

Departamento de Filosofía, Universidad de Málaga, Facultad de Filosofía y Letras Campus de Teatinos, E-29071 Málaga (España) 


\section{INTRODUCCIÓN}

EN LOS ALBORES DE LA MODERNIDAD, hubo un cambio metodológico en las ciencias y en la filosofía que determinó el desarrollo futuro de estas disciplinas. Los avances aportados por científicos como Copérnico, Kepler y Galileo daban a entender que no era necesario salvar la filosofía antigua a la hora de explicar los fenómenos empíricos y predecir el movimiento de los astros, es decir, no necesitaban esclarecer ningún tipo de metafísica a la hora de realizar esta tarea. Esto contrastaba enormemente con la práctica habitual hasta el momento, cuando los pensadores necesitaban esclarecer una metafísica que diese fundamento a toda explicación de los fenómenos empíricos. De este modo actuaba, por ejemplo, la física aristotélica. Sin embargo, dejando la metafísica a un lado y sin necesidad de apelar a ella, los nuevos científicos modernos se percataron de que podían presentar observaciones empíricas mucho más rigurosas que aquellas que habían realizado los antiguos. De hecho, en las universidades, lo habitual era el estudio de toda la filosofía y ciencia antigua como ciencia verdadera. Pero si atendemos al caso, por ejemplo, de la física aristotélica de astros perfectos, ésta no coincidía con los datos empíricos que recogían los modernos. Por ello, la rigurosidad de los filósofos griegos, tomada como el fundamento de todo quehacer científico durante casi dos mil años, quedaba cuestionada. Esta pérdida de autoridad de los filósofos antiguos y de la metafísica en general causó, por un lado, un fuerte desarrollo de las disciplinas científicas, que trabajaban ahora estableciendo solamente aquello que sus ojos podían ver, sin la necesidad de fundamentar el conocimiento más allá. Pero también causó, por otro lado, una separación casi definitiva de disciplinas, en la que la física podía actuar sin la metafísica, la ingeniería sin la ética y la mecánica sin la teología.

Esto, que comenzó a ponerse en práctica en la astronomía y física, tuvo también una fuerte influencia en la filosofía. Descartes, por ejemplo, levantó su sistema filosófico teniendo a las matemáticas como modelo, las cuales eran el fundamento de la nueva física y la nueva astronomía. La aplicación del modelo matemático, junto con la observación empírica, había demostrado su poder predictivo respecto a los movimientos de los cuerpos celestes, y Descartes intentó que ese poder predictivo, cuyo punto principal es la certeza que ofrece, se trasladase a la metafísica.

Poco a poco comenzaba a establecerse una rotura con la filosofía antigua. A pesar de ser el fundamento de toda práctica intelectual desde el siglo V a.C., la filosofía griega estaba dejando de servir como cimiento sobre el que construir explicaciones de la naturaleza, quedando relegada a un segundo plano. De hecho, toda filosofía comenzaba a tener valor solamente tras pasar por el tamiz de las observaciones empíricas o de la práctica matemática. Especialmente 
a partir del éxito de los Principia Mathematica de Newton, todo el saber no científico debía someterse a éste para ser comprendido como conocimiento verdadero y útil para el avance de las ciencias.

Por todo ello, la filosofía griega, aunque manteniendo su influencia en las humanidades, estaba perdiendo importancia en cuanto a sistematicidad para muchos de los autores modernos. Este es uno de los motivos por los cuales Descartes comienza su método filosófico de cero, sin necesidad de basarse explícitamente en Platón o Aristóteles, y además usando a la matemática como modelo a seguir en la metafísica.

Sin embargo, aunque la filosofía comenzaba a virar hacia el lado de los nuevos científicos, Leibniz es de los pocos que mantiene a la filosofía griega como fundamento sobre el que construir una filosofía. Primeramente, veremos cómo explícitamente Leibniz reconoce, al aceptar la necesidad de la interacción entre disciplinas, que las raíces de su filosofía están identificadas en Grecia. Además, la idea de que el mundo es susceptible de ser matematizado es una influencia claramente platónica, proveniente del Timeo. Y, por último, comprobaremos que cuando Leibniz intenta explicar la realidad mediante una sistematización de las verdades en una especie de cálculo, ejercicio al que hemos llamado maxima questio, observaremos que también existe una raíz griega que se deriva de la interacción de disciplinas y de esa matematización de la naturaleza.

De este modo, Leibniz comprende que la filosofía griega no debe ser obviada, sino absorbida para el avance de las ciencias, para explicar la realidad y, en definitiva, para responder a la maxima quaestio en filosofía que, para él, es presentar un sistema definitivo que responda a todas las cuestiones posibles.

\section{LA FILOSOFÍA ANTIGUA COMO PILAR DE LA FILOSOFÍA LEIBNIZIANA}

La magnitud de la figura de Leibniz es casi inabarcable, pues a día de hoy todavía no contamos con la edición completa de todos sus manuscritos. Sus escritos se separan en artículos, intercambios de cartas con unos 1500 correspondentes aproximadamente, libros, manuscritos, borradores y cientos de hojas todavía inéditas. Dada su imponente capacidad intelectual y su enorme producción científica, es difícil establecer ideas claras en algunos puntos concretos. Y debido a ello, las cartas juegan un papel primordial dentro de su corpus, pues los correspondentes ponen a prueba las tesis de Leibniz y él se ve en la obligación de contestar de un modo claro y adecuado. Por ello, el testimonio de Leibniz en sus cartas es esencial para su estudio, y es precisamente en su intercambio de cartas con Remond donde podemos comenzar a comprobar la importancia de la influencia griega en Leibniz. 
La carta del 10 de enero de 1714 de Leibniz a Remond ${ }^{1}$ es probablemente su carta más conocida. El motivo no es otro que contener la exposición más clara y precisa de la metafísica leibniziana, resumiendo por completo la finalidad de toda la producción filosófica y científica de Leibniz. De ahí el enorme valor que posee esta carta.

Afirma Leibniz en esta carta que, cuando contaba con tan solo 15 años, paseaba por un boscaje junto a la ciudad de Leipzig, donde nació y pasó su juventud, y tras acoger la filosofía de los mecanicistas, intentaba decidir si guardar o no las formas sustanciales, decidiendo finalmente guardarlas. ${ }^{2} \mathrm{La}$ actitud conciliadora de Leibniz, marcada fuertemente por su irenismo, ya está presente a sus tan sólo 15 años de edad, siempre que aceptemos la objetividad del Leibniz que escribe en 1714, en plena madurez, sobre sí mismo cuando tenía 15 años.

Primeramente prevaleció el mecanicismo, lo cual le llevó a aplicarse en matemáticas. Pero, sin embargo, una vez Leibniz comenzó a buscar las razones últimas del mecanicismo, acabó en la metafísica:

[...]Mas cuando buscaba las razones últimas del mecanicismo y de las leyes mismas del movimiento me sorprendió ver que era imposible encontrarlas en las matemáticas, y que había que volver a la metafísica. Esto me trajo de vuelta a las Entelequias, y de lo material a lo formal, y al final me hizo comprender, después de muchas correcciones y avances de mis nociones, que las Mónadas, o las sustancias simples, son las únicas sustancias verdaderas y que las cosas materiales no son más que fenómenos, aunque bien fundados y bien ligados. ${ }^{3}$

Pero no todo queda ahí. Esta unión entre los fenómenos bien fundados y las formas sustanciales, identificadas en Leibniz en sus famosas mónadas, fue visto anteriormente por Platón. Sin embargo, afirma Leibniz que los que han venido tras él no han sabido tomar el testigo y seguir esta enseñanza platónica adecuadamente. Vemos, por tanto, que el punto crucial de la filosofía leibniziana, que es la unión de la filosofía de los mecanicistas junto con las formas sustanciales, es una necesidad de la que Platón era consciente. Platón es consciente de que hay fenómenos que no pueden explicarse mecánicamente y en base a ello construye su sistema filosófico, pues la forma, no pudiendo ser explicada mecánicamente, constituiría el verdadero ser. Sin embargo, la visión platónica no podía ser acogida en su totalidad por Leibniz, pues existe una preeminencia de lo espiritual sobre lo material. Leibniz, sin embargo, y haciendo

1 Leibniz 1875-90, vol. III, p. 605-608.

2 Íbid., vol. III, p. 606.

3 Ídem. Las traducciones presentes en este artículo son propias, a no ser que se indique lo contrario. 
de nuevo gala de su actitud conciliadora, defiende que en la naturaleza no hay preeminencia de lo espiritual sobre lo material a la hora de estudiar la realidad de las cosas (aunque sí habría una mónada preeminente, la espiritual, sobre el resto de mónadas que constituyen el cuerpo de, en este caso, la persona). ${ }^{4}$

Sin embargo, hay equivocaciones que posee Platón y que comparte con Aristóteles, o al menos que poseen los platónicos (y aristotélicos) si seguimos con exactitud las palabras de Leibniz: que aunque están en lo cierto al buscar la fuente de las cosas en las causas final y eficiente, cometen dos errores. Primero, descuidar las causas eficientes y material (a las cuales Aristóteles sí da más importancia) y segundo, inferir que hay fenómenos naturales que no se pueden explicar mecánicamente. ${ }^{5}$ En el lado contrario se situarían los modernos, quienes promueven con exclusividad la filosofía mecanicista y quienes comenten el no menos grave error de rechazar todas las consideraciones metafísicas a la hora de explicar los fenómenos. Leibniz no puede, por tanto, acoger en su totalidad con las posiciones platónica, aristotélica ni mecánica. La suya es una opción original:

[...] todo se hace metafísicamente y mecánicamente al mismo tiempo en los fenómenos de la naturaleza, pero [...] la fuente de la mecánica está en la metafísica. No fue fácil descubrir este misterio, porque pocas personas se han molestado en unir estos dos tipos de estudio. ${ }^{6}$

Platón fue, sin duda, uno de ellos, como ya ha afirmado Leibniz. Pero en la modernidad él no fue el único en proponer esta unión entre el mecanicismo y la metafísica. Descartes fue probablemente el primer moderno en llevar a cabo esta difícil tarea. Y aunque revolucionó la filosofía con su método, afirma Leibniz que Descartes realizó la unión entre mecánica y metafísica no de un modo adecuado o suficiente, y dice que en ese sentido su filosofía es la antesala de la verdad. Lo que más ha detenido a Descartes para llegar a la Verdad es que ha ignorado las verdaderas leyes del movimiento. ${ }^{7}$ El gran fallo de la mecánica cartesiana es que, de hecho, las explicaciones sobre los fenómenos naturales no se corresponden con los datos que pueden recogerse a partir de la simple observación empírica. Por ello, sus explicaciones y predicciones son insuficientes y erróneas. El motivo de esto es sencillo: Descartes hipotecó la mecánica a la metafísica, y por ser la metafísica insuficiente, también lo fue la mecánica. Esto lo intenta solucionar Leibniz no dando prioridad a ninguno de

4 De ello habla Leibniz en Principes de la Nature et de la Grace, fondés en raison: Íbid, vol. VI, p. 604.

5 Íbid., vol. III, p. 607.

6 Ídem.

7 Ídem. 
esos dos ámbitos por encima del otro. Tanto la explicación mecánica por un lado tiene su importancia como la metafísica por otro. Para explicarlo todo, podría comenzarse tanto por la metafísica como por la física, y todas estas disciplinas nos llevarían por el camino de la Verdad. ${ }^{8}$

Hay otros científicos, afirma Leibniz, como Christiaan Huygens, ${ }^{9}$ que han desarrollado más adecuadamente sus estudios en mecánica, aunque en el caso de Huygens faltaría una metafísica que estuviese a la par en cuanto a importancia y desarrollo. ${ }^{10}$ Huygens de hecho fue un seguidor de Descartes desde joven, pues el filósofo francés era conocido de la familia y amigo de su padre, con quien mantuvo correspondencia, ${ }^{11}$ lo cual pudo dejar en Huygens una impresión importante. Su trabajo intelectual podría entenderse como una mejora de la mecánica cartesiana, pero no supo encontrar la unión entre mecánica y metafísica necesaria para poder explicar el mundo.

En definitiva, siguiendo las ideas de Leibniz podría decirse que nadie ha sabido resolver adecuadamente la maxima quaestio. Unos, mirando al cielo, se han olvidado de la tierra que pisan sus pies (Descartes, pero también Platón). Otros han puesto los pies en tierra, olvidándose de que más allá se encuentra un cielo que fundamenta lo existente en la tierra (Huygens y la mayoría de los científicos modernos).

\section{EL MUNDO ESCRITO EN CARACTERES MATEMÁTICOS}

La matematización del mundo es una noción muy extendida en la modernidad y que influencia en mayor o en menor grado a todos los intelectuales de la época. Esta importancia de la matemática proviene de la antigua Grecia, y de nuevo Platón es la influencia clave. Esta idea puede verse con claridad en el Timeo, cuya visión geométrica del mundo supone la base de la matematización que Galileo, Descartes y Leibniz proponen como metodológicamente esencial. Hasta tal punto es importante la figura de Platón respecto a la matematización en la modernidad, que Popper llegó a afirmar que, con el Timeo, Platón fue el fundador de la nueva ciencia. ${ }^{12}$

8 A veces Leibniz intenta llegar a la verdad mediante meditaciones metafísicas, como por ejemplo en su Monadología; en otras ocasiones, parte de trabajos científicos para alcanzar las verdades sobre la naturaleza de las cuales pueden deducirse principios verdaderos, tal y como puede comprobarse en muchas de las discusiones que mantiene con su maestro Huygens

9 Maestro de Leibniz en matemáticas en el periodo que éste pasó en París entre 16721674. Para más información véase Hofmann 1974.

10 De Huygens Leibniz llega a decir que «no tuvo gusto por la metafísica» Leibniz op. cit., vol. III, p. 607.

11 Accesible en Descartes 2009.

12 Popper 1999, p. 333. 
La matemática en la modernidad aparece como una especie de representación del mundo inteligible platónico. Los números como lenguaje del universo y tras el mismo universo serían aquello que da consistencia ontológica a la realidad física. Esta es la idea que parece deducirse de las famosas palabras de Galileo que afirman que el mundo está escrito en caracteres matemáticos:

La filosofía está escrita en ese libro enorme que tenemos continuamente abierto delante de nuestros ojos (hablo del universo), pero que no puede entenderse si no aprendemos primero a comprender la lengua y a conocer los caracteres con que se ha escrito. Está escrito en lengua matemática, y los caracteres son triángulos, círculos y otras figuras geométricas sin los cuales es humanamente imposible entender una palabra; sin ellos se deambula en vano por un laberinto oscuro. ${ }^{13}$

Leibniz, como moderno, no estuvo exento de esta influencia platónica. En su juventud y madurez el estatus epistemológico que poseen las matemáticas en las ciencias se encuentra intacto con respecto a 1623, cuando Galileo escribe las palabras citadas, y con respecto a 1637, momento en el que Descartes escribe su Discurso del Método. En el caso de Galileo, las matemáticas parecen suponer la esencia del mundo, los caracteres con los que las cosas son escritas y sin los cuales «se deambula en vano por un laberinto oscuro». En el caso de Descartes, el modelo matemático fundamenta a la metafísica, mostrándole los pasos que tiene que dar para alcanzar las verdades.

¿Y en el caso de Leibniz? A pesar de poseer las mismas influencias, su caso es totalmente distinto. Para él, la metafísica no debe estar subsumida al modelo matemático ni a la matemática en sí. Tampoco podremos comprender el universo mediante la matemática solamente, como en Galileo. Leibniz, contrariamente, supo ver la necesidad de que la matemática estuviese subsumida a la metafísica y no al contrario, en lo que puede parecer una vuelta al esquema platónico donde la metafísica tiene prioridad.

La preponderancia de la metafísica sobre la matemática, sin embargo, es actualmente una cuestión de debate en los círculos leibnizianos. El motivo es que la afirmación que hago, que hay en Leibniz una preponderancia de la metafísica sobre la matemática, parece contradecir una frase que dice Leibniz al Marqués de L'Hôpital el 27 de diciembre de 1694, la cual afirma «Mi metafísica es toda matemática por así decirlo, o se podría convertir». ${ }^{14}$ En ocasiones, se ha entendido que la matemática tendría un estatus similar al de la metafísica, pero Leibniz lo que parece querer decir no es que su metafísica es matemáticas, sino que su metafísica es matematizable, es decir, explicable mediante una especie de cálculo. En mi opinión, esa matematización de la metafísica en

13 Galilei 1979, p. 6.

14 Leibniz 1923ss, III 6, p. 253. 
Leibniz hace referencia a la máxima quaestio a la que nos venimos refiriendo en este artículo, ese sistema que al modo de un cálculo sería capaz de explicar todas las cuestiones posibles. Pero incluso ese sistema, aunque expresado matemáticamente en el sentido no de que sea matemática per se sino de que sea una especie de cálculo, sería de por sí una expresión metafísica del mundo. Quizá esta frase de Leibniz a L'Hôpital pueda entenderse mejor a la luz de otro lugar, en el que Leibniz afirma que toda su filosofía podría derivarse, de hecho, de unos cuantos axiomas. ${ }^{15}$

Por tanto, vemos que la puesta en marcha de esta maxima quaestio, su especificación y su formación se encuentra en la base de la idea platónica de la matematización de la naturaleza. Y aunque se encuentre esta idea en otros modernos como en Descartes, Leibniz es explícito de que sus fuentes son antes griegas que modernas como él mismo reconoce en su juventud:

[...] no temo afirmar que encuentro más cosas acertadas en los libros de la Física de Aristóteles que en las meditaciones de Descartes. Así de lejos me encuentro de ser cartesiano. Hasta me atrevería a añadir que la totalidad de estos ocho libros es admisible sin peligro para la filosofía reformada. ${ }^{16}$

Como podemos ver, no solamente encontramos indicios de influencia platónica en el sistema filosófico de Leibniz, sino que además hay señales claras de que la influencia aristotélica es más importante que aquella que proviene de los modernos, siendo muy relevante además que esto lo señale el mismo Leibniz en su juventud, cuando todavía se está formando intelectualmente y mantiene frescas sus primeras influencias:

[...] En efecto, lo que Aristóteles argumenta con respecto a la materia, la forma, la privación, la naturaleza, el lugar, lo infinito, el tiempo o el movimiento es en la mayoría de los casos algo cierto y demostrado. Incluso la forma sustancial, aquello por lo cual la sustancia de un cuerpo difiere de la de otro, ¿quién no la admitirá? Nada hay más cierto que la materia prima. Sólo se trata de comprobar una cosa: si lo que Aristóteles enunció de forma abstracta sobre la materia, la forma y el cambio, hay que explicarlo a través de la magnitud, la figura y el movimiento. ${ }^{17}$

Volviendo con el platonismo de Leibniz, encontramos más señales en extractos de cartas en las que sostiene que «La geometría es la más perfecta de las ciencias después de la metafísica» ${ }^{18}$ afirmación dirigida a su maestro Thomasius en 1668; o también en 1715, pocos meses antes de fallecer, cuando

15 Íbid., vol. I 19, p. 411.

16 Leibniz 1993, p. 99.

17 Ídem.

18 Íbid. p. 56. 
dice a Remond «Si alguien redujera a Platón a sistema, rendiría un gran servicio al género humano, y se vería que yo me acerco un poco a ello».. ${ }^{19}$ Ésta última cita es señalada por Bernardino Orio de Miguel para defender que Leibniz pasó su vida siendo un platónico que buscaba naturalizar su herencia griega:

[...] En efecto, y de momento sin más matizaciones, ser platónico significaba, para Leibniz, lo siguiente: todo cuanto cae bajo el ámbito de los sentidos no es en modo alguno lo real, de manera que una explicación adecuada de la naturaleza visible de las cosas debe necesariamente trascender ese territorio para buscar algún principio no material que lo funde, le dé unidad y sentido, lo haga inteligible. Pero «reducir a sistema» este pensamiento tan dual, tan evanescente, hacer descender las ideas eternas platónicas al reino de lo fungible y averiguar en qué consiste esa cosa no material, sino formal, que haga inteligible el mundo, pero sin estar fuera del mundo, requeriría para Leibniz «naturalizar» a Platón, volver al Aristóteles abandonado por los modernos purgándolo, al mismo tiempo, de algunas herencias escolásticas [...]. ${ }^{20}$

\section{LA MAXIMA QUAESTIO}

Si retomamos la carta de Leibniz a Remond del 10 de enero de 1714, veremos que Leibniz hace una declaración importante respecto al tema que nos ocupa. Afirma que, si hubiese estado menos distraído o que si fuese más joven, o que si tuviese la ayuda de jóvenes dispuestos, esperaría dar una manera de Spécieuse Generale, donde todas las verdades de razón serían reducidas a un tipo de cálculo. ${ }^{21}$ Esta es una de las formas que utiliza Leibniz para denominar lo que aquí hemos llamado maxima quaestio, un sistema general que pueda explicar todo como si de un cálculo se tratase. Con esta noción ocurre algo parecido como con la noción de Principio de Razón Suficiente, la cual se encuentra dispersa en todo el corpus leibniziano y explicada no solamente con distintas formulaciones y nombres, sino además con distinto valor, alcance y jerarquía con respecto a otros principios. ${ }^{22}$ Del mismo modo ocurre con la cuestión de un sistema definitivo de verdades, a veces formulada como si de un cálculo matemático se tratase, tal y como señala Leibniz a Remond, otras como de un lenguaje universal influenciado tanto por el lenguaje adánico como por el Cratilo de Platón, ${ }^{23}$ y otras como de una unión de todas estas cosas:

19 Leibniz 1875-90, vol. III, p. 637; Leibniz 2009ss, vol. 2, p. 241. También citado por Orio de Miguel 2013, p. 21.

20 Orio de Miguel op. cit., p. 21.

21 Leibniz 1875-90, vol. III, p. 605.

22 Nicolás 1993, p. 20.

23 Echeverría 1981, p. 114. 
[...] he comenzado a meditar ciertas consideraciones completamente nuevas, para reducir todos los razonamientos humanos a una especie de cálculo, que serviría para descubrir la verdad [...] esta especie de cálculo general proporcionaría al mismo tiempo una especie de escritura universal que tendría las ventajas de la de los chinos, porque cada cual la entendería en su lengua, pero que sobrepasaría infinitamente a la de los chinos porque cabría aprenderla en pocas semanas, ya que sus caracteres estarían ligados según el orden y la conexión de las cosas [...] esta misma escritura sería una especie de álgebra general y nos daría el medio para razonar calculando, de modo que en lugar de disputar podríamos decir: contemos. Y sucedería que los errores de razonamiento no serían sino errores de cálculo que se descubrirían mediante pruebas, como en la aritmética. Los hombres encontrarían en ella un juez de sus controversias verdaderamente infalible. ${ }^{24}$

Ya que este sistema definitivo se encuentra disperso igualmente en todo el corpus leibniziano sin una formulación final y definitiva y con distintas denominaciones, he decidido denominarlo como la máxima quaestio o la cuestión más importante, pues Leibniz parece presentarla como meta en su carrera a la hora de realizar cada una de las disciplinas en las que trabaja. Este sistema, tal y como hemos visto en los apartados anteriores, no podría existir de no haberse dado una importante influencia griega en Leibniz. Primero por la necesaria interacción entre disciplinas, especialmente la mecánica y la metafísica; y segundo, gracias a la idea de que la naturaleza es matematizable.

Aunque esta búsqueda de un sistema que respondiese a todas las cuestiones posibles se encuentra reflejada en toda la obra de Leibniz, él nunca llegó a ofrecer una formulación última. El funcionamiento preciso de este sistema, de hecho, no siempre queda expresado, así como el modo de interacción de las disciplinas que lo integran. Sin embargo, parece Leibniz afirmar, en el comienzo de la carta a Remond, que este sistema de verdades sería el siguiente paso que debería darse con respecto a la filosofía platónica:

Encuentro natural, Señor, que usted haya apreciado algo en mis pensamientos después de haber penetrado en los de Platón, autor de quien me acuerdo mucho, y que merecería ser sistematizado. Pienso poder llevar a la demostración las verdades que él no ha hecho más que progresar, y habiendo seguido sus trazas y las de otros grandes hombres, me halago de haber tenido beneficio y de haber alcanzado en cierto momento al menos, edita doctrina Sapientum templa serena ${ }^{25} .26$

24 Leibniz op. cit., vol. VII, p. 25-26. Traducción de Echeverría op. cit., p. 119-120.

25 «Nada hay más grato que ser dueño de los templos excelsos guarnecidos por el saber tranquilo de los sabios» Cita de Lucrecio. Traducción de Agustín García Calvo en Lucrecio 2010, Libro II, línea 8, p. 139.

26 Leibniz op. cit., vol. III, p. 605. 
Poco después de estas palabras afirma Leibniz que espera dar una Spécieuse Generale, tal y como he señalado. Por ello, creo que puede haber poco lugar para la duda al afirmar que Platón es una influencia esencial para la búsqueda de este sistema definitivo de verdades o maxima quaestio que buscaba realizar Leibniz como culminación de su filosofía.

\section{CONCLUSIÓN}

Aunque en la modernidad se comienza a cortar el cordón umbilical que une a los nuevos científicos con los filósofos griegos, Leibniz no solamente reconoce su influencia griega, sino que además ésta es esencial para el desarrollo de su sistema filosófico. Especialmente, hay varias nociones platónicas que facilitan la creación del sistema filosófico leibniziano, como son la interacción de disciplinas y la matematización del universo.

Según Leibniz, Platón vio la necesidad de unir una explicación mecánica de los fenómenos con aquella sobre las cosas que no pueden explicarse mecánicamente. En Leibniz esta idea está identificada en el uso de las formas sustanciales, que dará pie a su teoría de las mónadas. Para Leibniz, esta unión entre fenómenos bien fundados y las formas sustanciales es el único modo de crear una filosofía verdadera. A pesar de que Descartes fue el primer moderno en proponer una filosofía mecánica unida a la metafísica, al dar prioridad a la metafísica sobre la mecánica ocasionó la creación de una teoría mecánica que no se correspondía con lo observable empíricamente. Leibniz soluciona esto creando un sistema filosófico en el que tanto la metafísica como la observación de los fenómenos empíricos poseen la misma importancia a la hora de explicar la naturaleza.

Por otro lado, la matematización del mundo, idea que proviene del Timeo de Platón, permite a Leibniz poner en marcha la búsqueda de un sistema definitivo de verdades, como si de un cálculo matemático se tratase, estableciendo que los errores de razonamiento serían, de hecho, errores de cálculo. Por haberla formulado con distintas denominaciones y en distintos lugares, y por parecer la meta de la práctica filosófica y científica en el corpus leibniziano, la he denominado como la maxima quaestio.

De este modo, como el mismo Leibniz reconoce, su filosofía tiene un marcado carácter antiguo, en el sentido de que estaba influido por la filosofía de los griegos en un momento histórico en el que la práctica habitual de los filósofos y científicos era intentar comenzar desde cero. Quizá por eso, y porque el espíritu de los filósofos antiguos sigue presente entre todos aquellos que se dedican al quehacer filosófico, el pensamiento de Leibniz no se nos presenta como anticuado, sino como una filosofía que todavía hoy es relevante. 


\section{REFERENCIAS BIBLIOGRÁFICAS}

DESCARTES R. 2009: Tutte le lettere, 1619-1650, Milano: Bompiani.

ECHEVERRIA J. 1981: Leibniz. El autor y su obra, Barcelona: Barcanova.

GALILEI G. 1979: L'essayeur, París: Les Belles Lettres.

HOFMANN J.E. 1974: Leibniz in Paris (1672-1676). His growth to mathematical maturity, Cambridge: Cambridge University Press.

NICOLÁS J.A. 1993: Razón, verdad y libertad en G.W. Leibniz, Granada: Universidad de Granada.

LEIBNIZ G.W. 1875-90: Die philosophischen Schriften, C.I. Gerhardt (ed.), 7 vols, Berlín.

LEIBNIZ G.W. 1923ss: Sämtliche Schriften und Briefe, Deutschen Akademie der Wissenschaften zu Berlin (ed.), Berlín.

LEIBNIZ G.W. 1993: Correspondance Leibniz-Thomasius, Bodeüs R. (ed.), París: Vrin.

LEIBNIZ G.W. 2009ss: Obras Filosóficas y Científicas, Comares: Granada.

LUCRECIO 2010: De la naturaleza de las cosas, Madrid: Cátedra.

ORIO DE MIGUEL B. 2013: «Las fuentes científicas de Leibniz» en Arana J. (ed.), Leibniz y las ciencias, Madrid: Plaza y Valdés.

POPPER K. 1999: El mundo de Parménides. Ensayos sobre la ilustración presocrática, Barcelona: Paidós.

Miguel Palomo es doctorando en el Departamento de Filosofía, Lógica y Filosofía de la Ciencia de la Universidad de Sevilla

Lineas de investigación:

Mi intereses se centran en la filosofía de la naturaleza y la filosofía del siglo XVII. Más concretamente, actualmente investigo la correspondencia entre G.W. Leibniz y Christiaan Huygens, su maestro en matemáticas. Esta correspondencia nunca se ha traducido por completo ni ha sido objeto de un análisis sistemático, a pesar de la importancia de la figura de Huygens para el desarrollo del pensamiento filosófico y científico de Leibniz, y de que la mayoría de las discusiones entre ellos tuvieron lugar en estas cartas. Por un lado, me encuentro realizando este análisis sistemático de la correspondencia en forma de tesis doctoral; por otro, también me he embarcado en la tarea de realizar una edición crítica y la traducción de la totalidad de estas cartas al castellano para el volumen 17A de Obras Filosóficas y Científicas de G.W. Leibniz, volumen del que seré editor junto con el prof. Juan A. Nicolás (Universidad de Granada). Se espera, con este estudio y esta traducción, cubrir un importante hueco en el corpus de estudios leibnizianos.

Publicaciones recientes:

(2017, forthcoming) «Describing Reality: Bernoulli's Challenge of the Catenary Curve and its Mathematical Description by Leibniz and Huygens» en Leibniz and the Dialogue between 
Sciences, Philosophy and Engineering, 1646-2016. New Historical and Epistemological Insights, M. Fichant, A.R.E. Oliveira, P. Bussoti and R. Pisano (eds). London: College Publications.

(2016, coeditor) La monadología a debate. The Monadology of Leibniz to debate, Granada: Comares.

(2016) «Paths of Reason. Descartes in the Correspondence Between Leibniz and Huygens», en Ad felicitatem nostram alienamve. X. Internationaler Leibniz-Kongress, Wenchao Li (ed.), 6 Bände, Hildesheim/Zürich/New York: Olms, vol. 3 pp. 215-229.

Email: miguelpalomo@us.es 
